\title{
Time evolution of correlation functions and thermalization
}

\author{
Gian Franco Bonini周 and Christof Wetterich" \\ Institut für theoretische Physik, Universität Heidelberg, \\ Philosophenweg 16, Heidelberg 69120, Germany
}

July 27, 1999

\begin{abstract}
We investigate the time evolution of a classical ensemble of isolated periodic chains of $O(N)$-symmetric anharmonic oscillators. Our method is based on an exact evolution equation for the time dependence of correlation functions. We discuss its solutions in an approximation which retains all contributions in next-to-leading order in a $1 / N$ expansion and preserves time reflection symmetry. We observe effective irreversibility and approximate thermalization. At large time the system approaches stationary solutions in the vicinity of, but not identical to, thermal equilibrium. The ensemble therefore retains some memory of the initial condition beyond the conserved total energy. Such a behavior with incomplete thermalization is referred to as "mesoscopic dynamics". It is expected for systems in a small volume. Surprisingly, we find that the nonthermal asymptotic stationary solutions do not change for large volume. This raises questions on Boltzmann's conjecture that macroscopic isolated systems thermalize.
\end{abstract}

HD-THEP-99-23

\footnotetext{
*E-mail: bonini@thphys.uni-heidelberg.de

**E-mail: C.Wetterich@thphys.uni-heidelberg.de
} 


\section{Introduction}

A central piece in our understanding of the dynamics of large statistical systems is Boltzmann's conjecture that an ensemble of isolated interacting systems approaches thermal equilibrium at large times. The asymptotic values of the correlation functions can then be computed from the resulting microcanonical equilibrium ensemble. According to Boltzmann's conjecture their values only depend on the energy density of the system or, equivalently, the temperature (with standard modifications in the presence of other conserved extensive quantities). Besides the energy, all memory about the initial conditions is lost asymptotically - and also in practice if typical relaxation time scales are not too large. The thermalization conjecture only applies to spatially extended systems in the limit of infinite volume. So far no proof for this hypothesis has been given. Correspondingly, the question of how effective irreversibility arises from microscopic equations which are invariant under time reflection, or from the time reversible Liouville equation, has not found a complete answer todate. This effective irreversibility is the basis of widely used effective equations (like the Boltzmann equation).

We want to address two issues by a direct study of the time dependence of the correlation functions. The first concerns isolated systems with a finite number of degrees of freedom, corresponding to a finite volume $V$. No equilibration is expected for microscopic systems of only a few degrees of freedom. Recently, this has been demonstrated explicitly for the correlation functions 
of coupled anharmonic oscillators [1] 17. Let us assume for a moment that thermalization occurs for macroscopic systems. Then a smooth transition between the two extremes requires that there must be some intermediate volume or number of degrees of freedom where thermalization is incomplete, i.e. the ensemble retains some memory of the initial conditions. We may call the associated time evolution of the correlation functions "mesoscopic dynamics". Mesoscopic dynamics is characterized by partial effective irreversibility on one side, i.e. many details of the initial conditions get lost for asymptotic times. On the other hand, this "loss of memory" is not complete (as for strictly thermalizing systems) so that partial information about the initial state can be recovered even after an arbitrarily long time.

The second question concerns the validity of Boltzmann's conjecture for systems with a large volume. It is conceivable that thermalization remains incomplete even for macroscopic isolated systems. In this case some characteristics of mesoscopic dynamics would survive in the infinite volume limit. We emphasize that mesoscopic dynamics is always relevant in an appropriate volume range. Our second question therefore asks if and how the asymptotic loss of memory becomes complete as the volume becomes macroscopic. Within our approximations we find that certain features of mesoscopic dynamics remain present for isolated systems in the large volume limit!

Our investigation is based on an exact evolution equation for the time dependent effective action [2] which is the generating functional of the equal

\footnotetext{
${ }^{1}$ The issue would be completely different, of course, if the system were coupled to an external heat bath.
} 
time one particle irreducible (1PI) correlation functions. The direct study of the time evolution of the correlation functions circumvents the calculation of the time dependence of the probability distribution or density matrix. For classical systems the exact evolution equation is equivalent to the BBGKY hierarchy $[4$. A study of the 1PI correlation functions offers, however, new possibilities of systematic truncations. In particular, thermal equilibrium is now present as a stationary solution at every step of the truncation. Also the generalization to quantum statistics is straightforward and surprisingly simple [3]. An investigation of the general structure of the exact evolution equation for the time dependent effective action reveals many new stationary solutions besides thermal equilibrium [5]. The question of their dynamical role is part of the scope of this paper.

We will concentrate here on a particular example, namely a periodic chain of coupled anharmonic oscillators with $O(N)$ symmetry. The Hamiltonian

$$
H=\int_{0}^{l} d x\left[\frac{1}{2} p_{a}(x) p_{a}(x)+\frac{1}{2} q_{a}(x)\left(m^{2}-\Delta\right) q_{a}(x)+\frac{\lambda}{8 N}\left(q_{a}(x) q_{a}(x)\right)^{2}\right]
$$

describes an interacting time reversible system with microscopic time evolution given by

$$
\begin{aligned}
& \partial_{t} q_{a}(x)=p_{a}(x) \\
& \partial_{t} p_{a}(x)=-\left(m^{2}-\Delta\right) q_{a}(x)-\frac{\lambda}{2 N} q_{b}(x) q_{b}(x) q_{a}(x) .
\end{aligned}
$$


We may assume that the oscillators sit on discrete lattice sites with distance $a$ such that high momenta are cut off. Then $\Delta$ is an appropriate discretized Laplace operator involving neighboring sites. The index $a=1 \ldots N$ counts the oscillators at a given site and $H$ preserves $O(N)$ symmetry. Practical examples for small $N$ may be found in the form of ring-sized molecules with discrete translational symmetry along the ring. For example, the $q_{a}$ may describe displacements from the equilibrium position (with $p_{a}$ the associated momenta). The limit of large $l$ also describes large linear molecules if boundary effects from the ends can be neglected. Another interesting limit is $\lambda \rightarrow \infty$ for fixed $m^{2} / \lambda=-1$. This imposes the nonlinear constraint $q_{a} q_{a}=1$ and describes classical bosonic spin chains $(N=3$ for spin one). The length $l$ of the chain plays the role of the volume in three dimensional systems. For $l=0$ we are left with an ensemble of simple $N$-component anharmonic oscillators. This case has been studied in detail in [1]. No thermalization is possible since infinitely many conserved correlation functions keep the memory of the initial condition and obstruct thermalization. The conserved cumulants are simply related to powers of the conserved energy and squared $O(N)$-angular momentum $L^{2}$, i.e. $\left\langle E^{r}\left(L^{2}\right)^{s}>\right.$ does not change in time for arbitrary $r$ and $s$. If Boltzmann's conjecture is true, asymptotic thermalization governs the behavior for $l \rightarrow \infty$. In this case we conclude that there must be a range of $l$ with mesoscopic dynamics, describing the transition between the limits $l \rightarrow 0$ and $l \rightarrow \infty$. On the other hand, if 
Boltzmann's conjecture does not hold for isolated systems of this type some features of mesoscopic dynamics are expected to be relevant also for $l \rightarrow \infty$.

Our system is also a prototype for classical and quantum field theories. From this point of view it describes a one-dimensional $O(N)$-symmetric scalar field theory. There is no conceptual problem in its generalization to three dimensions where it would be relevant for cosmology (e.g. inflation and parametric resonance or dynamical scalar fields playing a role in late cosmology), for particle physics (e.g. pions in heavy ion collisions) or for many systems in statistical mechanics.

Although most practical applications are for small $N$, we also discuss the limit of large $N$. The reason is that one of our truncation schemes is a systematic expansion in powers of $1 / N$. The leading order in the $1 / N$ expansion has been discussed by various groups with different methods [6], [5]. In this first approximation infinitely many conserved quantities preclude thermalization [5]. We include here all contributions in next to leading order in $1 / N$. In particular, this includes scattering in three dimensional field theories. Particle numbers for individual momentum modes are no longer conserved and there is no immediately visible obstruction to thermalization anymore.

We concentrate in this paper on classical statistics. This has the advantage that our results can easily be compared with other methods. In particular, it should be feasible to solve the microscopic equations numerically with given initial values and then take averages over an ensemble of 
initial values. In this way the equal time correlation functions discussed in this paper can be directly measured at any later time. The generalization to quantum statistics is straightforward in our approach and will be postponed to a subsequent paper.

We investigate homogeneous (translation invariant) and $O(N)$ symmetric ensembles. Individual members of these ensembles do not, of course, share this high degree of symmetry. For generic initial conditions the solution of the microscopic evolution equation (2) is highly inhomogeneous and shows no $O(N)$ symmetry. The high symmetry of the ensemble only means that we weight the initial conditions according to a probability distribution that exhibits this symmetry. In practice, there is actually no need to specify the probability distribution at the initial time $t_{0}$ explicitely. It is often more effective to specify the correlation functions at $t_{0}$. These will constitute the initial data for our differential flow equations. In the present paper we mainly consider gaussian initial perturbations from equilibrium, where all 1PI $n$-point functions except the two-point functions are equal to their thermal values.

We find effective irreversibility as a property of the solutions of our time reversible flow equation. In a wider sense this is due to the existence of attractive fixed point solutions. In our context an attractive fixed point does not necessarily mean that all solutions for a given class of initial ensembles asymptotically reach this fixed point. The characteristic behavior for large $t$ 
is rather characterized by high-frequency oscillations of the correlation functions around time averaged stationary mean values. The approach to the stationary behavior for the time averaged correlation functions shows three characteristic features:

- First we find a fast initial irreversible behavior on typical microscopic time scales between the inverse momentum cutoff $\Lambda^{-1}=a / \pi$ (with $a$ the lattice distance) and the inverse mass $m^{-1}$. An example is given in Fig 11 where we plot the time evolution of the ratio between kinetic energy and total energy for an out-of-equilibrium system. Comparison with the leading order in the $1 / N$ expansion reveals no qualitative difference. We conclude that this first period of irreversibility is not related to scattering but rather described by dephasing [6], [5]. A "rough thermalization" takes already place at this very early stage.

- The first stage of rapid "rough thermalization" does not bring the twopoint functions near the equilibrium values. In Fig.2 we display the evolution of the time-averaged two-point function $B(q)$ which characterizes the gaussian part of the probability distribution for the momenta $p_{a}(q)=\int d x e^{-i q x} p_{a}(x)$ by $<p_{a}(q) p_{b}\left(q^{\prime}\right)>=2 \pi \delta\left(q+q^{\prime}\right) \delta_{a b} G^{\pi \pi}(q)$, $G^{\pi \pi}(q)=B^{-1}(q)\left(1-C^{2}(q) /(A(q) B(q))\right)^{-1}$ (see below). In thermal equilibrium one expects the Maxwell velocity distribution with $B(q)=$ $\beta=1 / T$ independent of $q$ and $C(q)=0$. One observes that an initially disturbed $B(q)$ approaches a stationary value only on time scales much 
larger than $m^{-1}$. "Scattering" is essential for this aspect of irreversibility. This can be seen by a comparison with the leading $1 / N$ behavior where $B(q)$ oscillates around a time independent value for every $q$. In leading order $1 / N$ no energy is exchanged between the different Fourier modes. This explains why time averaged values for $B(q)$ are stationary from the beginning and therefore cannot equilibrate.

- The exchange of energy between different Fourier modes in next-toleading order in the $1 / N$ expansion drives the time averaged velocity distribution toward a stationary value. It may be a surprise that in general this stationary value differs from thermal equilibrium with uniform $B(q)=\beta$. The implications of this finding will be discussed in the last section.

\section{The method}

Our investigation is based on the time-dependent effective action [2], which generates the equal-time 1PI correlation functions. We consider an $N$-component $(1+1)$-dimensional scalar $\phi^{4}$ theory and ensembles which are invariant under internal $O(N)$ transformations, spatial translations, and reflection. Our truncation retains all 1PI $n$-point functions up to $n=4$ and omits 1 PI vertices with $n \geq 6$. One should notice that this still includes connected $n$-point functions with arbitrary $n$. In this approximation the effective action of our model becomes: 

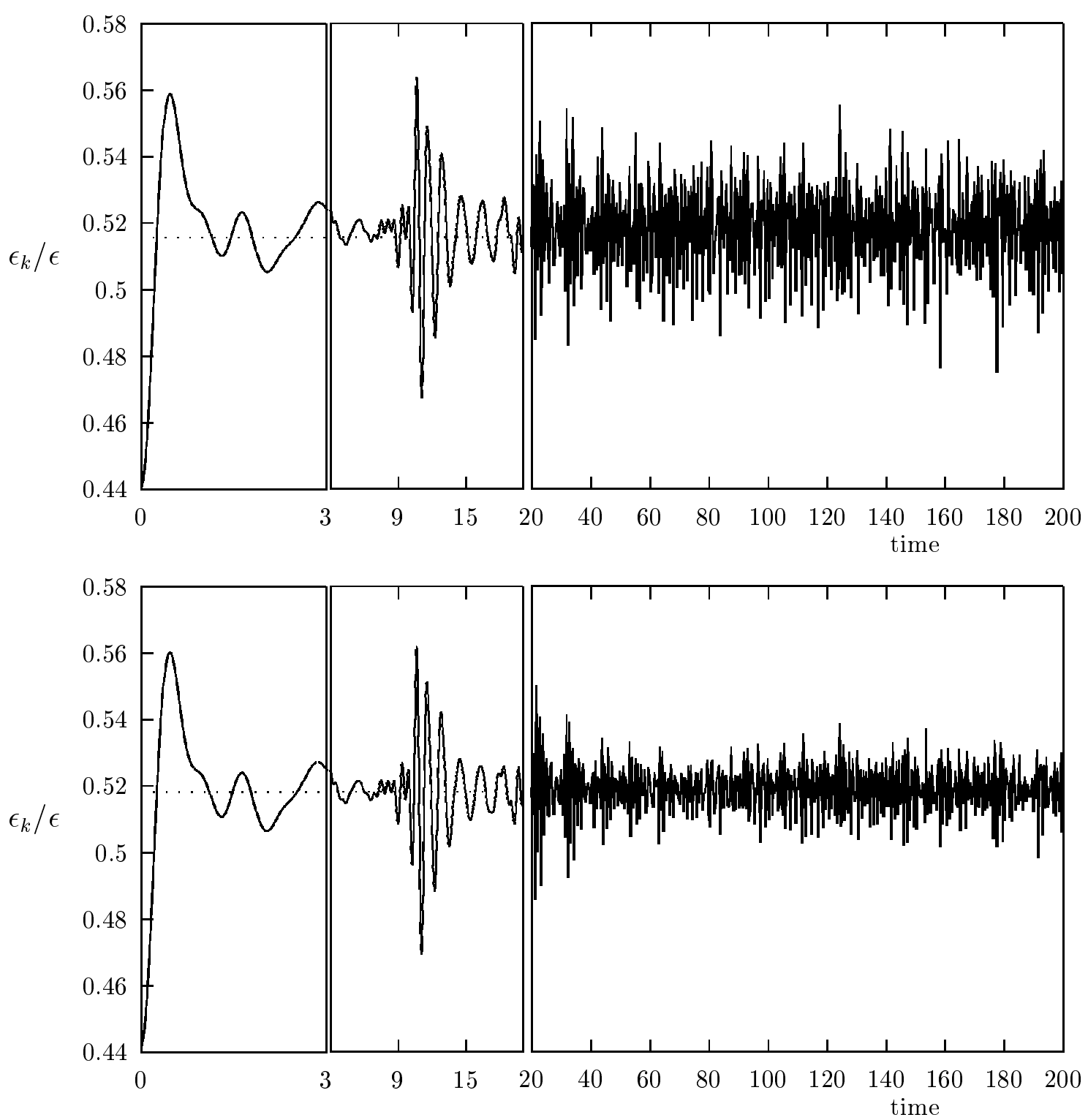

Figure 1: Energy "equipartition". We show $\epsilon_{k} / \epsilon$ as a function of time, in the leading (upper panel) and next-to-leading $1 / N$ approximations, for $\lambda=2$, $N=20, \Lambda=5$, and $l=20.1$. The system is initially displaced from equilibrium according to a gaussian perturbation in $B(q)\left(\beta=0.5, D_{B}=\right.$ $0.25, \Delta_{B}=0.469, q_{B}=2.5$; cf. Eq.(16)). Horizontal lines correspond to thermal equilibrium for $T=2$. 


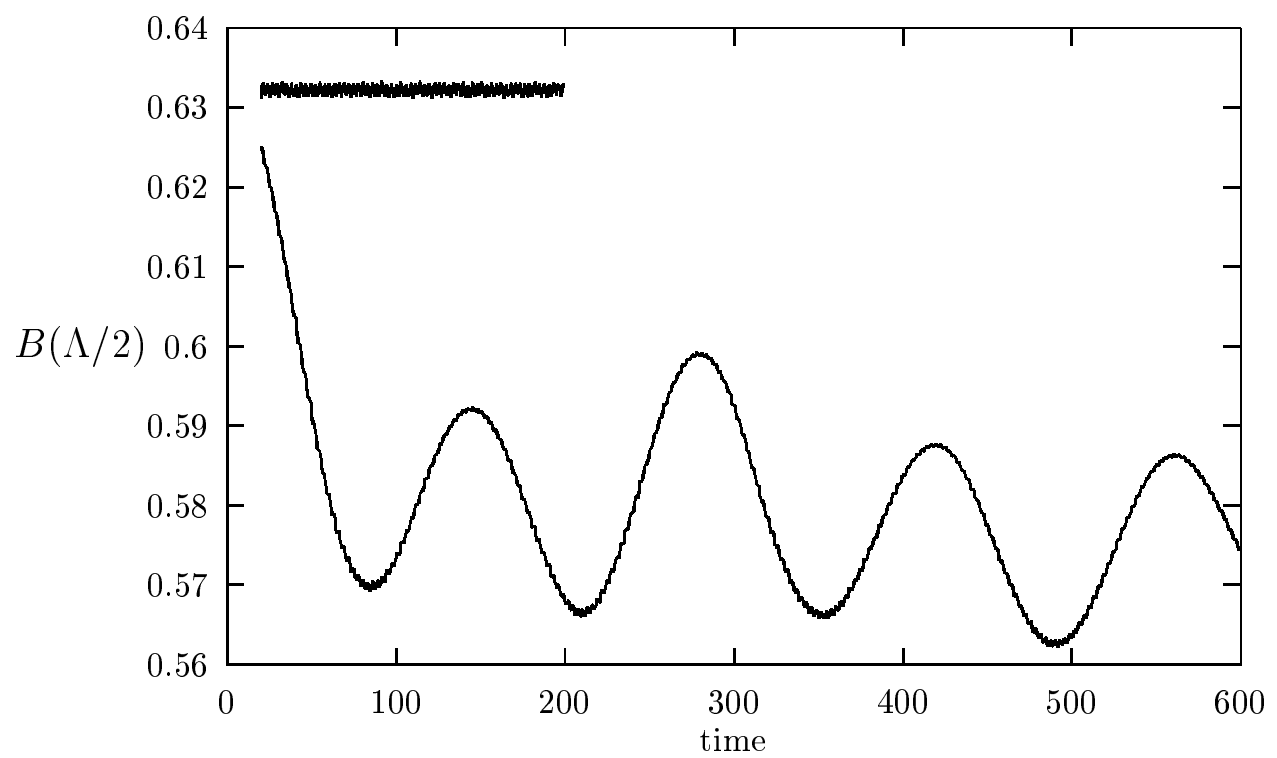

Figure 2: Time evolution of the two-point correlation function. We plot $B(\Lambda / 2)$ for the same system as in Fig.1, in the leading (upper curve) and next-to-leading $1 / N$ approximations. The plotted values are averages over the time interval $[t-20, t]$. Note that the equilibrium value for the corresponding energy is $B(\Lambda / 2)=0.5$. In the leading $1 / N$ approximation $B(q)$ stays esssentially constant for arbitrarily long time. 


$$
\begin{aligned}
\Gamma[\phi, \pi ; t]= & \frac{1}{2} \int \frac{d^{D} q}{(2 \pi)^{D}}\left\{A(q) \phi_{a}^{*}(q) \phi_{a}(q)+B(q) \pi_{a}^{*}(q) \pi_{a}(q)\right. \\
& \left.+2 C(q) \pi_{a}^{*}(q) \phi_{a}(q)\right\}+\frac{1}{8 N} \int \frac{d^{D} q_{1}}{(2 \pi)^{D}} \frac{d^{D} q_{2}}{(2 \pi)^{D}} \frac{d^{D} q_{3}}{(2 \pi)^{D}} \\
& \left\{u\left(q_{1}, q_{2}, q_{3}\right) \phi_{a}\left(q_{1}\right) \phi_{a}\left(q_{2}\right) \phi_{b}\left(q_{3}\right) \phi_{b}\left(-q_{1}-q_{2}-q_{3}\right)\right. \\
& +v\left(q_{1}, q_{2}, q_{3}\right) \pi_{a}\left(q_{1}\right) \phi_{a}\left(q_{2}\right) \phi_{b}\left(q_{3}\right) \phi_{b}\left(-q_{1}-q_{2}-q_{3}\right) \\
& +w\left(q_{1}, q_{2}, q_{3}\right) \pi_{a}\left(q_{1}\right) \pi_{a}\left(q_{2}\right) \phi_{b}\left(q_{3}\right) \phi_{b}\left(-q_{1}-q_{2}-q_{3}\right) \\
& +s\left(q_{1}, q_{2}, q_{3}\right)\left[\pi_{a}\left(q_{1}\right) \pi_{b}\left(q_{2}\right) \phi_{a}\left(q_{3}\right) \phi_{b}\left(-q_{1}-q_{2}-q_{3}\right)\right. \\
& \left.\quad-\pi_{a}\left(q_{1}\right) \pi_{a}\left(q_{2}\right) \phi_{b}\left(q_{3}\right) \phi_{b}\left(-q_{1}-q_{2}-q_{3}\right)\right] \\
& +y\left(q_{1}, q_{2}, q_{3}\right) \pi_{a}\left(q_{1}\right) \pi_{a}\left(q_{2}\right) \pi_{b}\left(q_{3}\right) \phi_{b}\left(-q_{1}-q_{2}-q_{3}\right) \\
& \left.+z\left(q_{1}, q_{2}, q_{3}\right) \pi_{a}\left(q_{1}\right) \pi_{a}\left(q_{2}\right) \pi_{b}\left(q_{3}\right) \pi_{b}\left(-q_{1}-q_{2}-q_{3}\right)\right\}
\end{aligned}
$$

where the "couplings" $A(q), u\left(q_{1}, q_{2}, q_{3}\right)$, etc. depend on time. The 1PI $n$ point functions are obtained by taking derivatives of $\Gamma$ with respect to $\phi$ and $\pi$, the second derivative being the inverse propagator. For example, the connected two-point function for $q_{a}$ reads

$$
<q_{a}(x) q_{b}(y)>_{c}=G(x-y) \delta_{a b}=\int \frac{d^{D} q}{(2 \pi)^{D}} G(q) e^{i q(x-y)} \delta_{a b},
$$

where

$$
G(q)=\frac{B(q)}{A(q) B(q)-C^{2}(q)} .
$$

The time evolution of $\Gamma$ induced by Eq.(2) is dictated by the nonlinear evolution operator 


$$
\partial_{t} \Gamma[\phi, \pi ; t]=-\left(\mathcal{L}_{\mathrm{cl}}+\mathcal{L}_{\mathrm{q}}\right) \Gamma[\phi, \pi ; t]
$$

where $\left(\psi_{i} \equiv\left(\phi_{a}, \pi_{a}\right)\right)$

$$
\begin{aligned}
\mathcal{L}_{\mathrm{cl}}= & \int d^{D} x\left\{\pi_{a}(x) \frac{\delta}{\delta \phi_{a}(x)}+\phi_{a}(x)\left(\nabla^{2}-m^{2}\right) \frac{\delta}{\delta \pi_{a}(x)}\right. \\
& -\frac{\lambda}{2 N}\left[\phi_{b}(x) \phi_{b}(x) \phi_{a}(x)+\phi_{a}(x) G_{b b}^{\phi \phi}(x, x)+2 \phi_{b}(x) G_{b a}^{\phi \phi}(x, x)\right. \\
& -\int d^{D} x_{1} d^{D} x_{2} d^{D} x_{3} G_{a i}^{\phi \psi}\left(x, x_{1}\right) G_{b j}^{\phi \psi}\left(x, x_{2}\right) \\
& \left.\left.\times G_{b k}^{\phi \psi}\left(x, x_{3}\right) \frac{\delta^{3} \Gamma}{\delta \psi_{i}\left(x_{1}\right) \delta \psi_{j}\left(x_{2}\right) \delta \psi_{k}\left(x_{3}\right)}\right] \frac{\delta}{\delta \pi_{a}(x)}\right\},
\end{aligned}
$$

and

$$
\mathcal{L}_{\mathrm{q}}=\frac{\lambda}{8 N} \hbar^{2} \int d^{D} x \phi_{a}(x) \frac{\delta \Gamma}{\delta \pi_{b}(x)} \frac{\delta \Gamma}{\delta \pi_{b}(x)} \frac{\delta}{\delta \pi_{a}(x)}
$$

with

$$
\left[G^{-1}\right]_{a b}^{\psi \psi^{\prime}}(x, y)=\frac{\delta^{2} \Gamma}{\delta \psi_{a}(x) \psi_{b}^{\prime}(y)},
$$

and $G$ of (5) corresponding to $G^{\phi \phi}$.

The exact flow equations for the two-point functions follow from taking the second derivatives of Eq.(6) with respect to $\phi$ and $\pi$ at $\phi=\pi=0$ :

$$
\begin{aligned}
\dot{A}(q) & =2 \omega^{2}(q) C(q) \\
\dot{B}(q) & =-2 C(q)-\frac{2}{N} \gamma(q) B(q) \\
\dot{C}(q) & =-A(q)+\omega^{2}(q) B(q)-\frac{\gamma(q)}{N} C(q),
\end{aligned}
$$


where

$$
\begin{aligned}
& \omega^{2}(q) \equiv q^{2}+m^{2}+\lambda \frac{(N+2)}{2 N} \int_{p} G(p) \\
& -\frac{\lambda(N+2)}{8 N^{2}} \int_{q_{1}, q_{2}} G\left(q_{1}\right) G\left(q_{2}\right) G\left(q-q_{2}-q_{1}\right) \cdot \\
& {\left[4 u\left(q_{1},-q, q_{2}\right)-c\left(q_{1}\right) c\left(q_{2}\right) c\left(q-q_{1}-q_{2}\right) y\left(q-q_{1}-q_{2}, q_{1}, q_{2}\right)\right.} \\
& -c\left(q_{1}\right)\left[2 v\left(-q_{1},-q_{2}, q\right)+v\left(-q_{1}, q,-q_{2}\right)\right] \\
& \left.+2 c\left(q_{2}\right) c\left(-q_{1}-q_{2}+q\right) w\left(q-q_{1}-q_{2}, q_{2}, q_{1}\right)\right] \\
& \gamma(q) \equiv \frac{\lambda(N+2)}{8 N} \int_{q_{1}, q_{2}} G\left(q_{1}\right) G\left(q_{2}\right) G\left(q-q_{2}-q_{1}\right) \cdot \\
& {\left[v\left(q,-q_{1},-q_{2}\right)-4 c\left(q_{1}\right) c\left(q_{2}\right) c\left(q-q_{1}-q_{2}\right) z\left(-q_{1}, q,-q_{2}\right)\right.} \\
& -2 c\left(q_{1}\right) w\left(-q_{1}, q,-q_{2}\right) \\
& \left.+c\left(q_{2}\right) c\left(q-q_{1}-q_{2}\right)\left[y\left(q-q_{1}-q_{2}, q_{2},-q\right)+2 y\left(-q, q_{1}, q-q_{1}-q_{2}\right)\right]\right] \\
& c(q) \equiv \frac{C(q)}{B(q)}
\end{aligned}
$$

Similarly, the flow equation for the quartic coupling $u$ reads ?:

$$
\begin{aligned}
\dot{u}\left(q_{1}, q_{2}, q_{3}\right)= & {\left[\omega^{2}\left(q_{1}\right) v\left(q_{1}, q_{2}, q_{3}\right)+4 \lambda C\left(q_{1}\right)-4 \lambda C\left(q_{2}\right)\left(S_{1}\left(q_{1}+q_{2}, q_{3}\right)\right.\right.} \\
& \left.\left.+S_{2}\left(q_{2}+q_{3}, q_{1}\right)\right)-\lambda \hbar^{2} C\left(q_{1}\right) C\left(q_{2}\right) C\left(q_{3}\right)\right]_{S Y M}
\end{aligned}
$$

\footnotetext{
${ }^{2}$ We display here only one of the six flow equations for the 4-point couplings. The remaining five equations can be found in Appendix A.
} 
where the subscript $S Y M$ implies symmetrization with respect to the appropriate permutations of $q_{1}, q_{2}, q_{3}$ and $q_{4}=-\left(q_{1}+q_{2}+q_{3}\right)$. Here we have introduced the momentum integrals

$$
\begin{aligned}
S_{1}\left(q_{1}, q_{2}\right) \equiv & \frac{1}{2 N} \int_{q} G(q) G\left(q+q_{1}\right) \cdot \\
& {\left[(N+2) u\left(q+q_{1},-q, q_{2}\right)+2 u\left(q_{2},-q, q+q_{1}\right)\right.} \\
& -\frac{1}{2} c(q)\left((N+2) v\left(-q, q+q_{1}, q_{2}\right)+2 v\left(-q, q_{2}, q+q_{1}\right)\right) \\
& +\frac{1}{2} c\left(q+q_{1}\right) c(q)\left(( N + 2 ) \left(w\left(-q, q+q_{1}, q_{2}\right)-\frac{1}{2} s\left(-q, q+q_{1}, q_{2}\right)\right.\right. \\
& \left.\left.\left.-\frac{1}{2} s\left(q+q_{1},-q, q_{2}\right)\right)+s\left(-q, q+q_{1}, q_{2}\right)\right)\right] \\
S_{2}\left(q_{1}, q_{2}\right) \equiv & \frac{1}{2 N} \int_{q} G(q) G\left(-q-q_{1}\right)\left[4 u\left(-q, q_{2}, q+q_{1}\right)\right. \\
& +c(q) c\left(-q-q_{1}\right) s\left(q+q_{1},-q,-q_{1}-q_{2}\right) \\
& \left.-c(q)\left(v\left(-q, q_{2}, q+q_{1}\right)+v\left(-q,-q_{1}-q_{2}, q+q_{1}\right)\right)\right] .
\end{aligned}
$$

The flow equations for the quartic couplings $u$, $v$, etc. are not exact since we have truncated the contributions from 1PI 6-point functions. We furthermore have omitted the two-loop contribution to the evolution of the quartic couplings. Our approximation may be viewed as the second order in a weighted loop expansion where the evolution of every 1PI $2 m$-point function is computed in $\left(n_{L}+1-m\right)$-loop order (i.e. two loops for the two-point function, one loop for the four-point function). It is easy to convince oneself that this expansion retains systematically all contribution in order $N^{1-n_{L}}$. In our case it also includes (incompletely) terms of order $1 / N^{2}$. For comparison 
we employ a second systematic expansion, namely the $1 / N$ expansion, where all terms of order $1 / N^{2}$ are omitted in the flow equations.

The flow equations conserve exactly the energy density $\epsilon=E / l$ :

$$
\begin{aligned}
\epsilon= & \frac{N}{2} \int_{q}\left\{B^{-1}(q)+G(q)\left[q^{2}+m^{2}+c^{2}(q)+\frac{N+2}{4 N} \lambda \int_{p} G(p)\right]\right\} \\
& -\frac{N+2}{8 N} \lambda \int_{q_{1}, q_{2}, q_{3}} G\left(q_{1}\right) G\left(q_{2}\right) G\left(q_{3}\right) G\left(q_{4}\right)\left[u\left(q_{1}, q_{2}, q_{3}\right)-v\left(q_{1}, q_{2}, q_{3}\right) c\left(q_{1}\right)\right. \\
& +w\left(q_{1}, q_{2}, q_{3}\right) c\left(q_{1}\right) c\left(q_{2}\right)-y\left(q_{1}, q_{2}, q_{3}\right) c\left(q_{1}\right) c\left(q_{2}\right) c\left(q_{3}\right) \\
& \left.+z\left(q_{1}, q_{2}, q_{3}\right) c\left(q_{1}\right) c\left(q_{2}\right) c\left(q_{3}\right) c\left(q_{4}\right)\right]
\end{aligned}
$$

whereas the squared $O(N)$ "angular momentum" density

$$
\begin{aligned}
& \frac{L^{2}}{l}=N(N-1) \int_{q_{1}} G\left(q_{1}\right) B^{-1}\left(q_{1}\right) . \\
& \left\{1-\frac{1}{4 N} \int_{q_{2}} G\left(q_{2}\right) B^{-1}\left(q_{2}\right)\left(2 w\left(q_{1}, q_{2},-q_{2}\right)-2 s\left(q_{1}, q_{2},-q_{2}\right)-s\left(q_{1}, q_{2},-q_{1}\right)\right)\right\}
\end{aligned}
$$

is conserved only up to relative corrections of order $1 / N^{2}$. Additional independent conserved quantities of the form $<E^{r}\left(L^{2}\right)^{s}>-<E>^{r}<\left(L^{2}\right)>^{s}$ are suppressed by inverse powers of $N$. They are not conserved by the truncated equations. The kinetic energy density $\epsilon_{k}=\frac{N}{2} \int_{q}\left\{B^{-1}(q)+G(q) c^{2}(q)\right\}$ is, of course, not separately conserved.

We have solved the classical flow equations $(\hbar=0)$ numerically for a discretized system with $N_{l}$ points and an ultraviolet cutoff $\Lambda=$ a few times $m$, using a standard fourth-order Runge-Kutta algorithm which has the property

\footnotetext{
${ }^{3}$ With the exception of the subleading contributions to the evolution of the 4-point couplings that are contained in $\omega^{2}$ and $\gamma$. These have to be retained in order to ensure exact energy conservation.
} 
of being exactly time-reversible. We only consider here positive $m^{2}$ and set the mass scale by $m=1$. For a typical cutoff $\Lambda=5$ and $N_{l}=32$, the length of the chain is $l=N_{l} a=\pi N_{l} / \Lambda \simeq 20.1$.

\section{$3 \quad$ Equilibrium properties}

As a first step in the numerical analysis, we compute the classical thermal equilibrium configuration (defined by the conditions: $C=v=w=s=y=$ $z=0, B=\beta)$ for different values of the parameters. In our approximation this corresponds to the solution of the Schwinger-Dyson equations for $A$ and $u$ that follow from the requirement $\partial_{t} \Gamma=0$. For $\lambda / N \ll 1$ it is possible to derive the thermal values of $A$ and $u$ iteratively as power series in $\lambda / N$. In general, however, this method fails, and we found it simpler to use the flow equations themselves, starting from the $N \rightarrow \infty$ thermal fixed point, letting the system evolve for a while $\left(\Delta t>m^{-1}\right)$, taking time averages of the correlation functions, adjusting them in accordance with the thermal fixed point constraints, and repeating the procedure until stationary behavior with the desired accuracy was obtained. We were thus able to obtain configurations that were thermal to a very good approximation $(\Delta B / B<0.001)$. In Fig. 3 we display the energy density and the squared angular momentum as functions of the temperature. We also show the frequency $\omega_{e q}(0)$ which is related in equilibrium to a (partially) renormalized temperature-dependent mass by $\omega_{e q}^{2}(q)=A_{e q}(q) T=T / G_{e q}(q)=m_{R}^{2}+Z(q) q^{2}$. 

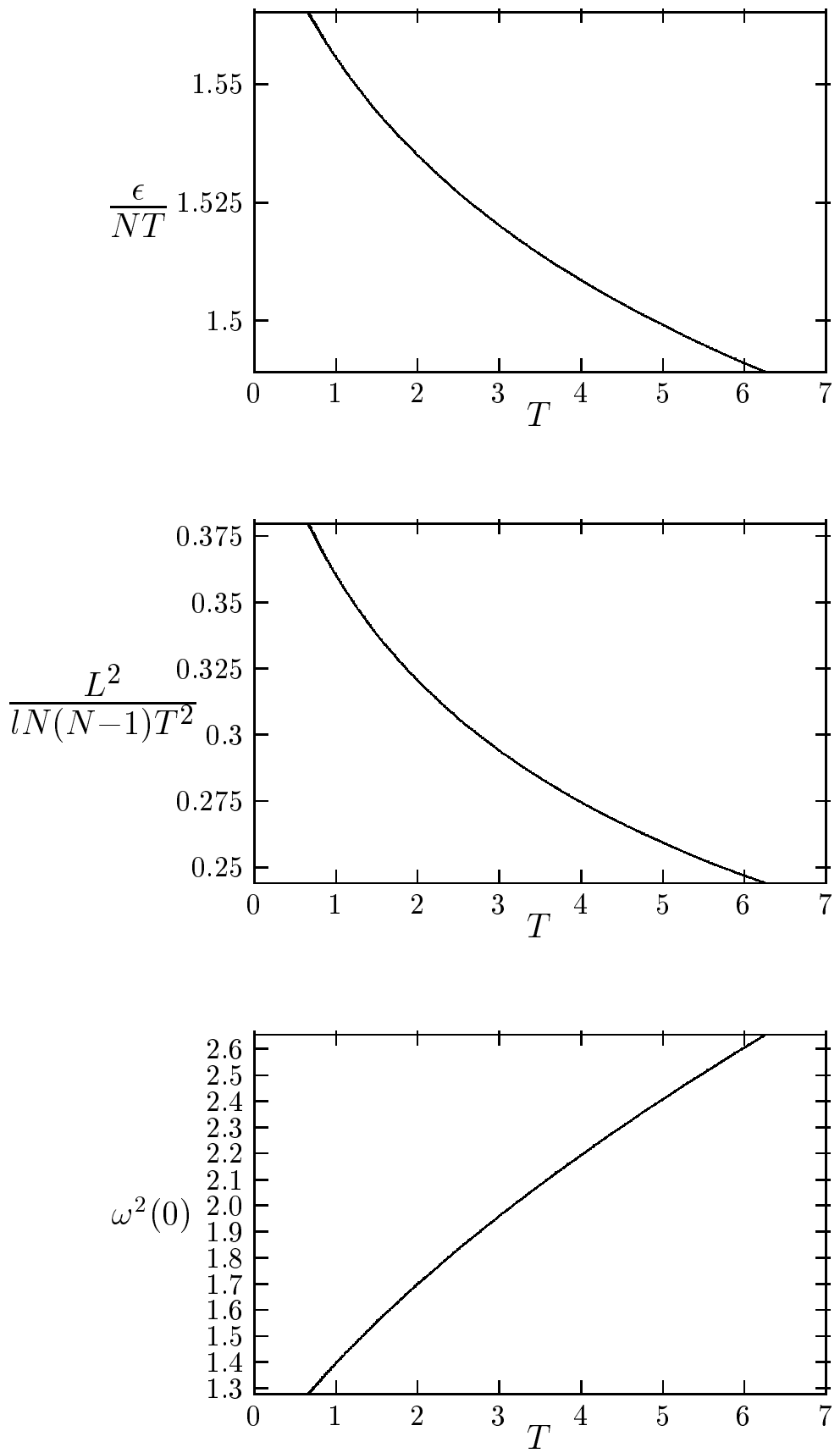

Figure 3: Thermodynamic equilibrium properties. We show $\epsilon / N T$, $L^{2} /\left(l N(N-1) T^{2}\right)$, and $\omega^{2}(0)$ as functions of $T$, for a system with $N=20$, $\lambda=2, \Lambda=5$, and $l=20.1$. 


\section{Dephasing and scattering}

Next we discuss the evolution of ensembles that are initially not in thermal equilibrium. We opted for gaussian perturbations from the thermal state with initial two-point functions:

$$
\begin{aligned}
& A_{0}(q)=C_{A}\left(A_{e q}(q)+D_{A}\left[e^{-\frac{\left(q-q_{A}\right)^{2}}{\Delta_{A}^{2}}}+e^{-\frac{\left(q+q_{A}\right)^{2}}{\Delta_{A}^{2}}}\right]\right) \\
& B_{0}(q)=C_{B}\left(\beta+D_{B}\left[e^{-\frac{\left(q-q_{B}\right)^{2}}{\Delta_{B}^{2}}}+e^{-\frac{\left(q+q_{B}\right)^{2}}{\Delta_{B}^{2}}}\right]\right) \\
& C_{0}(q)=D_{C}\left[e^{-\frac{\left(q-q_{C}\right)^{2}}{\Delta_{C}^{2}}}+e^{-\frac{\left(q+q_{C}\right)^{2}}{\Delta_{C}^{2}}}\right] .
\end{aligned}
$$

The constants $D_{A}, D_{B}, D_{C}, q_{A}, q_{B}, q_{C}, \Delta_{A}, \Delta_{B}, \Delta_{C}$ are arbitrary, whereas $C_{A}$ and $C_{B}$ are tuned so that the perturbed system has the same $E$ and $L^{2}$ as the unperturbed thermal equilibrium ensemble. We also use superpositions of gaussian perturbations with the property that the initial deviations $D_{A}$, $D_{B}$ for small and large $q^{2}$ are small.

In order to assess the importance of "scattering" for the equilibration of different physical quantities, we first compare the results obtained by using the full equations (10)-(12) with those obtained by keeping only the leading terms in $1 / N$ (i.e. neglecting all 4 -point functions). We repeat here that in leading order $1 / N$ scattering is absent and only kinetic dephasing can induce a smoothening and averaging out of the perturbation.

As we can see in Fig.11, even in the absence of interactions, energy equipartition is achieved to a very good approximation. Also $\omega(0)$ equilibrates approximately (Fig. (4). The individual correlation functions $A(q)$ and $B(q)$, 
however, do not equilibrate in the absence of scattering (Figs.22, 5). They oscillate around constant values. When the effect of the time-dependent four-point functions is added the picture changes. We now see that the original perturbations in the correlation functions are damped and smoothened out by the evolution, although they do not reach exact thermal equilibrium. In Fig.6 we show the time evolution of $A(0)$ and $A(\Lambda / 2)$. We remind that $\sqrt{T A(0)}$ should approach $\omega(0)$ in thermal equilibrium.

\section{Asymptotic behavior and thermalization}

We have seen in the previous section that next-to-leading order terms in the weighted loop or $1 / N$ expansions induce an energy exchange between different Fourier modes, which is a prerequisite for thermalization. Also "particle numbers" for individual Fourier modes are no longer conserved separately. Because of this energy exchange, a system with nonthermal initial conditions is driven towards thermal equilibrium. At late times its correlation

functions oscillate around mean values that are "more thermal" than in the case of mere dephasing. In Fig.7 we show the evolution of the time-averaged correlation functions $A(q)$ and $B(q)$ in a typical case. One clearly observes the initial approach towards the equilibrium values. For large $t$, however, stationary values are reached which deviate from the equilibrium correlations. These stationary values correspond to exact fixed points of the truncated evolution equations. We have computed the fixed points by methods similar to the computation of the equilibrium. They are also displayed in Fig.7. 

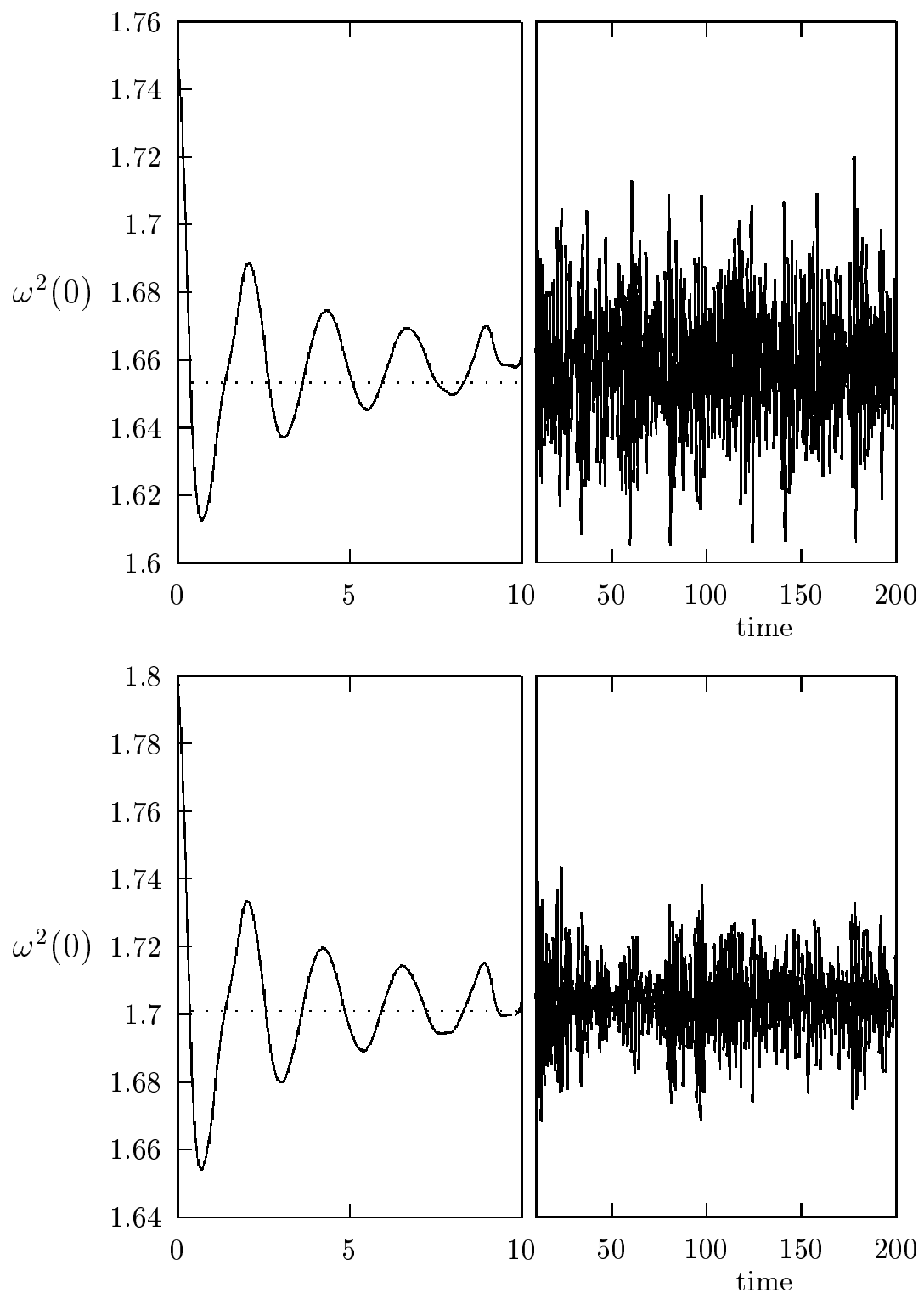

Figure 4: Evolution of the frequency $\omega^{2}(0)$, in the leading (upper) and nextto-leading $1 / N$ approximations (same parameters as in Figs.1-2). Comparison with the equilibrium value (also shown) indicates the "more thermal" behavior due to the inclusion of scattering, see also Fig. 3 for $T=2$. 

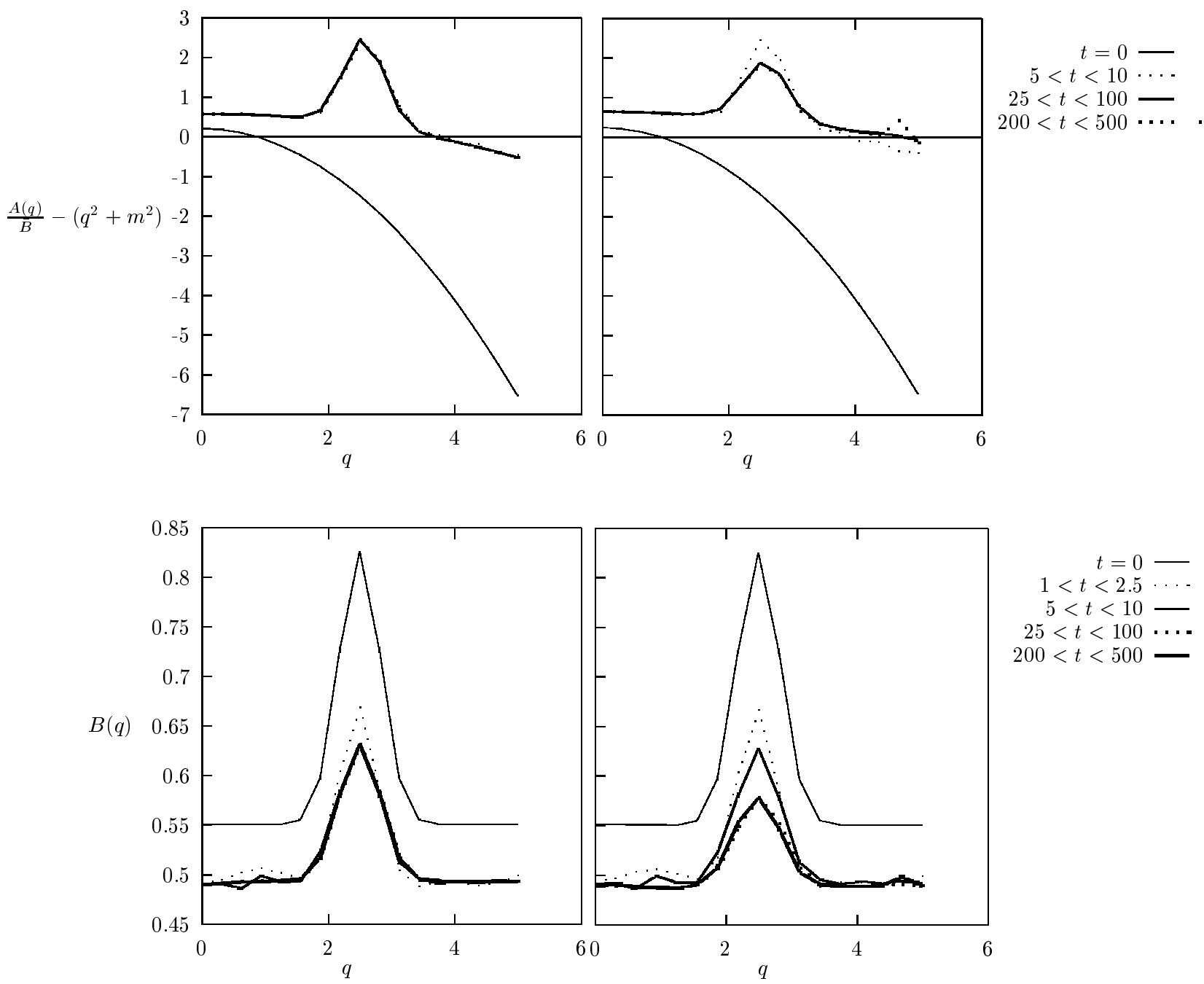

Figure 5: A comparison between the leading-order (left) and next-to-leadingorder (right) evolutions of the two-point correlation functions. The first row shows $A(q) /\left(\sum_{q^{\prime}} B\left(q^{\prime}\right) / N_{l}\right)-\left(q^{2}+m^{2}\right)$, which is a measure for the deviation of the inverse propagator from the classical value. The second row gives $B(q)$, or the deviation from the Maxwell velocity distribution $B(q)=\beta$. The correlation functions are averaged over various time intervals. The initial values at $t=0$ are also shown. 

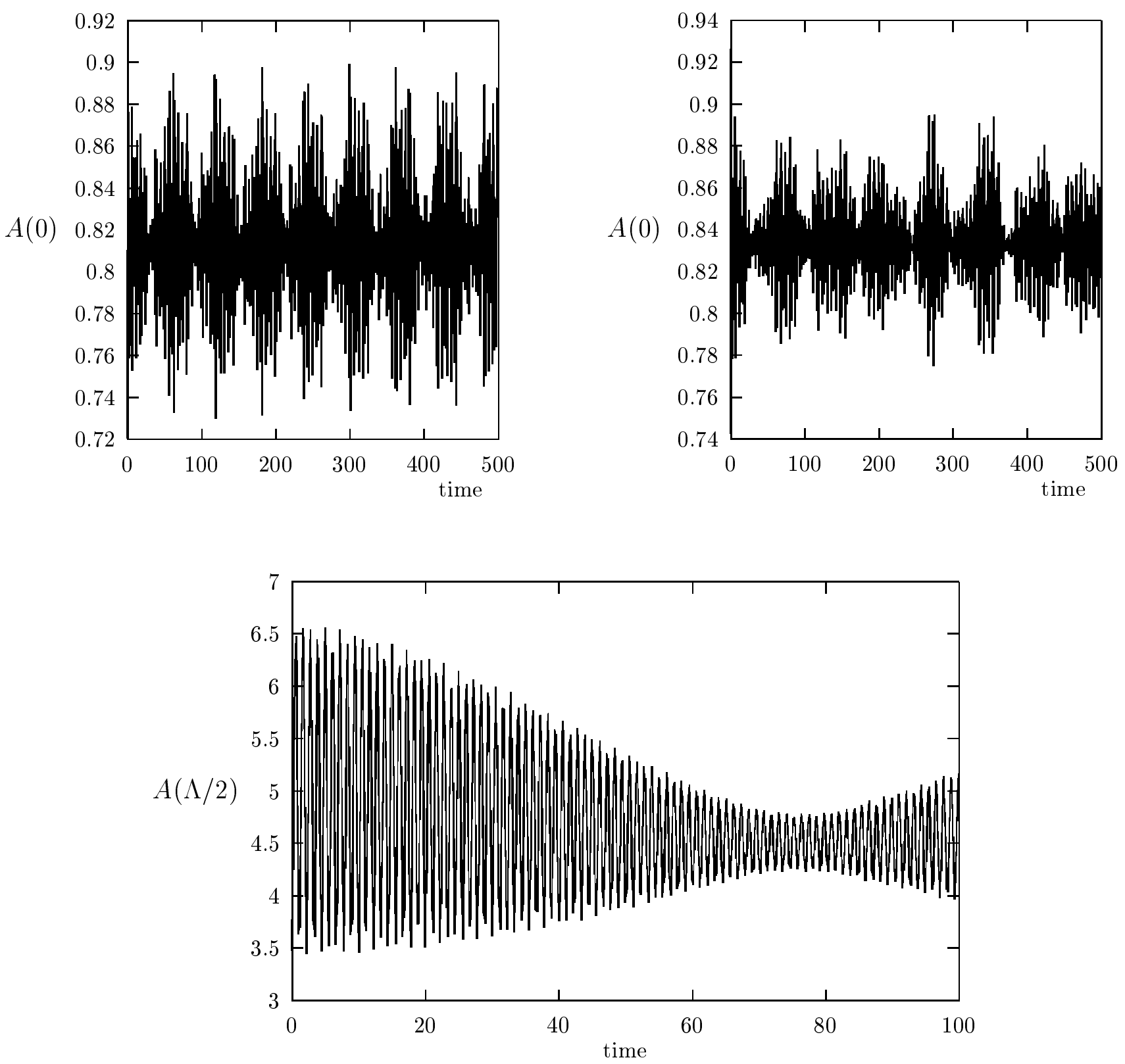

Figure 6: Full time evolution of the two-point functions $A(0)$ (both leading and next-to-leading order) and $A(\Lambda / 2)$ (next-to-leading only), without averaging. 
We notice that the effect of the deviation from thermal equilibrium on the correlation function in coordinate space is small, as can be seen from the plot of $G(x-y)$.

In Table 1 we collect the asymptotic stationary values for several different choices of physical parameters and temperature, and otherwise identical initial conditions the $10 \%$ level for not too small values of $\lambda / N$. As a general rule, the larger $\lambda / N$, the faster the system approaches its asymptotic limit, and the closer this limit is to the thermal values. In order to save on computer time, we have therefore opted for rather small values of $N(1<N<5)$ and values of $\lambda$ between 1 and 60. Of course, for small $N$ and/or large $\lambda / N$ the applicability of a $1 / N$ or weigthed-loop expansion is questionable. There is no satisfactory way to assess the reliability of either truncation in the strong coupling regime. However, we feel comforted by the fact that the 4-point functions never grow so large as to make the system unstable, and that the time fluctuations of $L^{2}$ (which we remind is conserved exactly by the exact evolution but only up to relative corrections $\sim 1 / N^{2}$ by the truncated equations) are always quite small (at the $1 \%$ level for $\lambda=60, N=1$ ). The most direct test seems to be a comparison between the results from the weighted-loop and the $1 / N$ expansions. For $N=1$, large $\lambda$ (Table $1 \mathrm{~b}$ ), the two truncations lead to very different large time stationary values, and it is therefore conceivable

\footnotetext{
${ }^{4}$ This holds up to discretization corrections, since we define identical initial conditions by identical continuous functions of $q$.
} 
that the asymptotic departure from thermal equilibrium is due to truncation errors. As $N$ gets larger and $N$ smaller, however, the two expansions agree much better (Fig.8) and it is less plausible that higher order $1 / N$ corrections could account for the asymptotic departures from equilibrium. We therefore believe that our numerical results support the existence of nonthermal attractive fixed points also for the exact (i.e. non-truncated) system. The implications of this claim are discussed in the next section.

As a final word of caution, we should consider the possibility that full thermalization does occur even for small $\lambda / N$, but on longer time scales than those we have been able to probe. This issue can be settled only with further, more computer-intensive investigations. What we have seen so far does not support this hypothesis. Even if that turned out to be the case, our method would still be very useful for identifying the various time scales (dephasing partial thermalization - complete thermalization) and for assessing the role of thermalization in practical problems, where extremely large time scales are not always relevant.

We have also studied the volume dependence of the large time behavior for a particular choice of parameters (Table 1c, Fig.99). Apparently, the large volume limit still differs from thermal equilibrium. Relatively small volumes $\left(l \sim 20 m^{-1}\right)$ seem often to be sufficient for an extrapolation to infinite volume.

Finally, we have investigated a limited sample of initial conditions that are not symmetric under time reversal, and whose backward and forward 
evolutions therefore differ in their microscopic details. In all cases, the large time asymptotic averaged values of the correlation functions come out the same in both time directions.

\begin{tabular}{|c|c|c|c|c|c|}
\hline$\lambda$ & $N$ & $\beta$ & $l$ & $\frac{B(\Lambda / 2)-\beta}{\beta}$ & $\frac{A(\Lambda / 2)-A_{e q}(\Lambda / 2)}{A_{e q}(\Lambda / 2)}$ \\
\hline (*) 3 & 1 & 0.3 & 20.1 & $0.083 \pm 0.005$ & 0.08 \\
\hline (*) 1 & 2 & 0.3 & 20.1 & $0.158 \pm 0.005$ & 0.16 \\
\hline (*) 1 & 5 & 0.3 & 20.1 & $0.16 \pm 0.01$ & 0.16 \\
\hline 10 & 1 & 0.3 & 20.1 & $0.02 \pm 0.005$ & 0.03 \\
\hline (*) 10 & 1 & 0.3 & 20.1 & $0.03 \pm 0.005$ & 0.03 \\
\hline 60 & 3 & 0.3 & 20.1 & $0.07 \pm 0.01$ & 0.07 \\
\hline 60 & 1 & 0.3 & 20.1 & $-0.013 \pm 0.003$ & 0.013 \\
\hline (*) 60 & 1 & 0.3 & 20.1 & $0.008 \pm 0.003$ & 0.009 \\
\hline$(*) 1$ & 3 & 0.3 & 5.03 & $0.23 \pm 0.003$ & 0.23 \\
\hline (*) 1 & 3 & 0.3 & 10.05 & $0.20 \pm 0.015$ & 0.20 \\
\hline (*) 1 & 3 & 0.3 & 15.08 & $0.183 \pm 0.015$ & 0.24 \\
\hline (*) 1 & 3 & 0.3 & 17.6 & $0.18 \pm 0.005$ & 0.18 \\
\hline 1 & 3 & 0.3 & 17.6 & $0.187 \pm 0.006$ & 0.185 \\
\hline$(*) 1$ & 3 & 0.3 & 20.1 & $0.11 \pm 0.03$ & 0.12 \\
\hline 1 & 3 & 0.3 & 20.1 & $0.167 \pm 0.008$ & 0.17 \\
\hline (*) 1 & 3 & 0.3 & 25.1 & $0.167 \pm 0.017$ & 0.16 \\
\hline
\end{tabular}

Table 1: Asymptotic displacement from thermal equilibrium for different $\lambda, N$, $\beta$ and $l$. For all configurations $\Lambda=5$. The initial perturbation is a superposition of three gaussians with $D_{B}=\beta / 2, q_{B}=\Lambda / 2, \Delta_{B}=1.5 \Lambda / 16 ; D_{B}=-\beta / 4$, $q_{B}=5 \Lambda / 16, \Delta_{B}=1.5 \Lambda / 16 ;$ and $D_{B}=-\beta / 4, q_{B}=11 \Lambda / 16, \Delta_{B}=1.5 \Lambda / 16$. Configurations marked by $(*)$ are evolved according to the $1 / N$ expansion, the others according to the weighted loop expansion. For the first entries (a) the 
$1 / N$ expansion seems reliable. The second group of entries (b) concerns large interactions with a rapid approach to asymptotic behavior. Finally, the last entries (c) are used for a study of the volume dependence, see also Fig.9.

\section{Discussion}

Our study of the evolution equations, applied to various initial nonthermal probability distributions, clearly establishes effectively irreversible behavior. This is not put in by hand in the form of irreversible evolution equations. Our equations are manifestly invariant under time reflection. Effective irreversibility is rather related to the existence of stationary solutions or fixed points towards which the flow is effectively attracted. It can be observed by evolving in time directions $t \rightarrow \pm \infty$. We see both the effects of dephasing, i.e. effective loss of phase information, and scattering, i.e. energy exchange between different momentum modes. Our investigations are carried out for translation invariant ensembles such that the energy exchange is not merely due to a classical background field evolving in time. The inclusion of scattering effects is a crucial step beyond the leading $1 / N$-approximation used in the past [6]. Genuinely, the system approaches asymptotically for large $t$ an oscillatory behavior of the correlation functions around a stationary solution. The time averaged values of the correlation functions are close to the corresponding stationary solutions. In a rough sense, the stationary so-

lutions share many properties of thermal equilibrium. The corresponding fixed points are, nevertheless, not identical to the thermal fixed point. The 

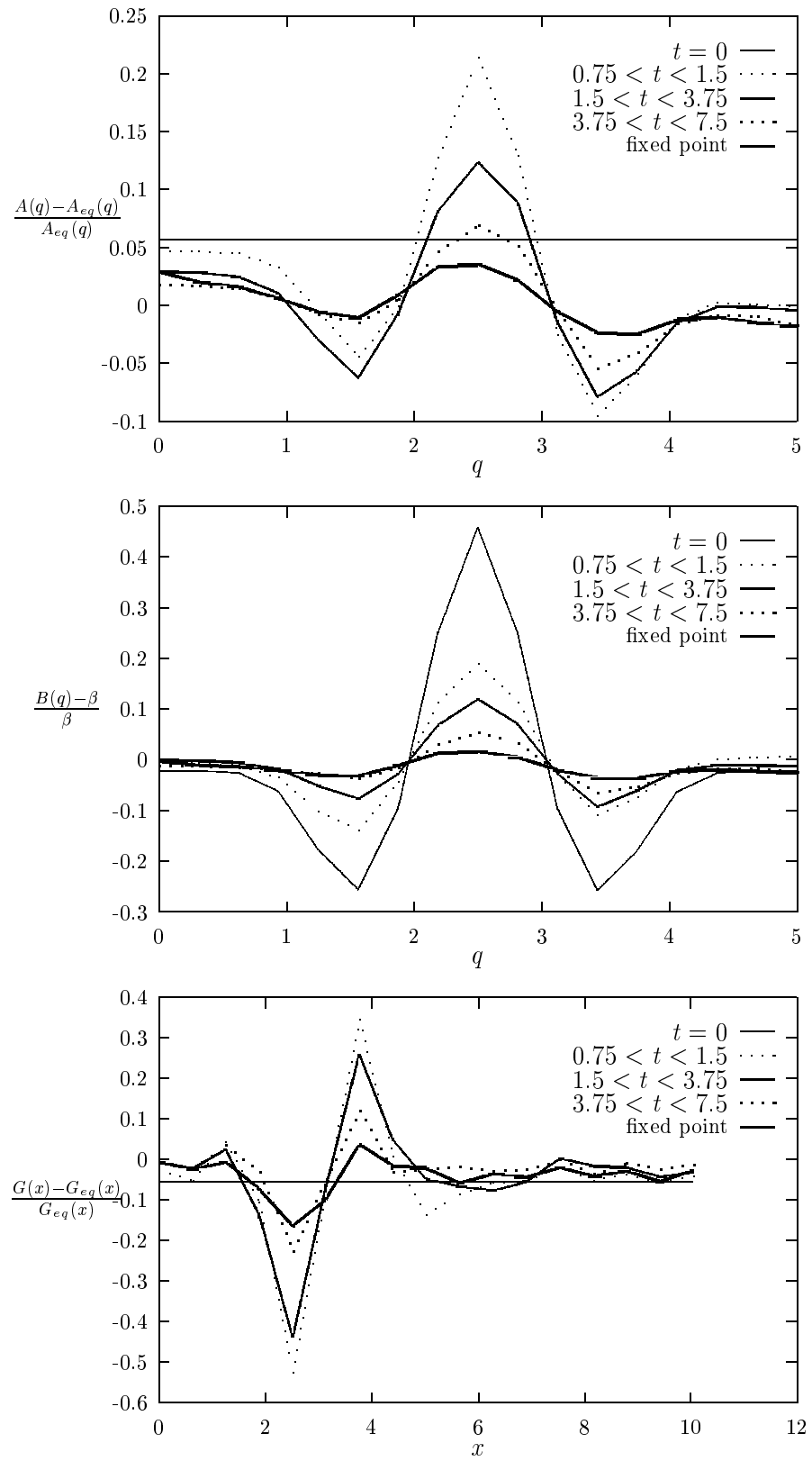

Figure 7: Time evolution of the two-point functions for a system with $\lambda=10$, $N=1, \beta=0.3$, with nonthermal initial conditions. The three panels show, from top to bottom, the relative deviations of $A(q), B(q)$ and $G(x)$, averaged over various time intervals, from their respective thermal values. 


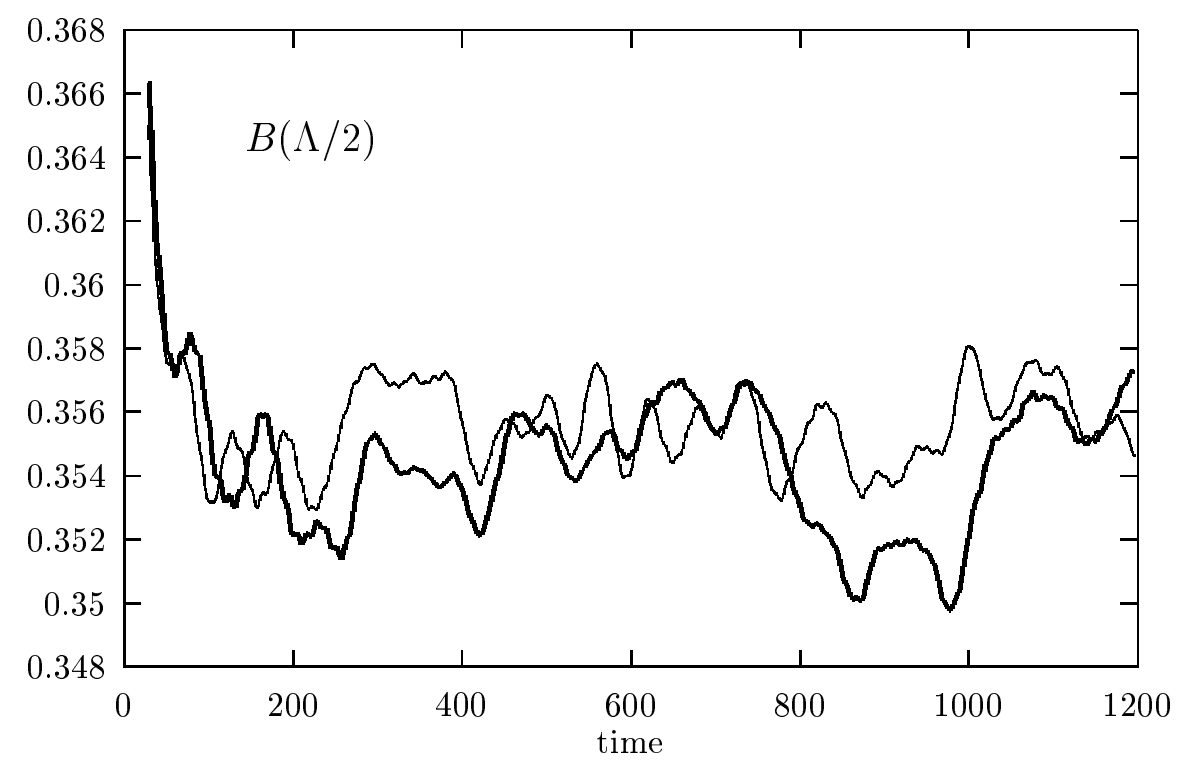

Figure 8: Time evolution of $B(\Lambda / 2)$ averaged over $\Delta t=30$. The parameters are $\lambda=1, N=3, \beta=0.3, l=17.6$, and the initial perturbation is described in the the caption of Table 1 . The two curves correspond to the $1 / N$ and weighted loop expansions (bold and plain, respectively). There is no sign or an asymptotic approach to the precise thermal value $B(\Lambda / 2)=0.3$. 


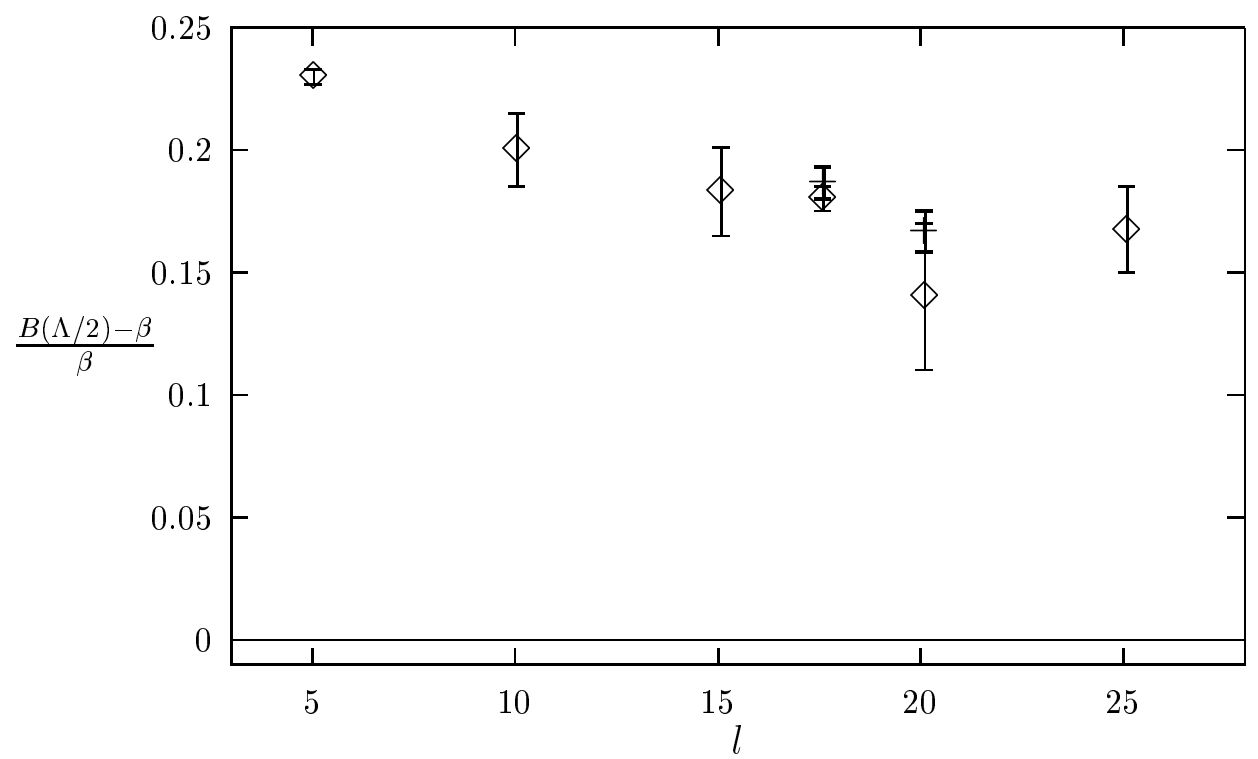

Figure 9: Volume dependence. We show the asymptotic time-averaged value of $B(\Lambda / 2)$ as a function of $l$, for the system of Table 1c. Diamonds and crosses correspond to the $1 / N$ and weighted loop expansions, respectively. 
latter turns out to be a point in a whole manifold of fixed points. For generic fixed points in this manifold the correlation functions differ from thermal equilibrium.

Perhaps the most interesting observation concerns the difference of the asymptotic stationary ensembles from the thermal ensemble. The system retains memory of the initial conditions beyond the energy density or temperature. Isolated systems seem to differ in this respect from systems coupled to a heat bath. Arguments why isolated systems do not thermalize exactly can be given on different levels. First there are exact obstructions from conserved correlation functions. An example is the squared angular momentum density $<L^{2}>/ l$. In thermal equilibrium this quantity can be computed as a function of temperature, $L_{e q}^{2} / l=N(N-1) T \int_{q} G_{e q}(q ; T)$. Since $<L^{2}>$ is conserved by the exact flow equations, any initial value of $\left\langle L^{2}>\right.$ different from the thermal one implies immediately that the correlation functions appearing in Eqs.(14)-(15) cannot all take thermal values for $t \rightarrow \infty$, not even in a time-averaged sense. We emphasize that this obstruction is based on an exactly conserved quantity and therefore cannot be an artifact of insufficient approximations.

In principle, one could take care of the conservation of $\left\langle L^{2}\right\rangle$ by an extension of the thermodynamic description, adding a chemical potential for $L^{2}$. The problem is, however, that there exist infinitely many conserved combinations of correlation functions. Another prominent example is the global "specific heat" $c_{V}=<(E-<E>)^{2}>/ T^{2} l$ which corresponds 
again to an exactly conserved combination of correlation functions $<(E-<$ $E>)^{2}>=\frac{N l}{2} \int_{q} G_{\pi \pi}^{2}(q)+\ldots$ Also, the additional "chemical potentials" would multiply nonlocal expressions like $E^{2}=\int d x d y \epsilon(x) \epsilon(y)$. Indeed, the probability distribution $p \sim e^{-b(E / l) E}$ is stationary for an arbitrary function $b(\epsilon)$. The Boltzmann distribution $b(\epsilon)=\beta$ is only a special case. If the function $b(\epsilon)$ is not constant the value of $c_{V}$ typically differs from the one in thermal equilibriump. These general considerations hold for an arbitrary number of space dimensions. They show that for isolated systems there cannot be a proof of strict thermalization using arguments of ergodicity. Strict thermalization for arbitrary initial conditions is in contradiction with the existence of conserved combinations of correlation functions].

On a second level one observes the existence of a large manifold of fixed points or stationary solutions for the truncated flow equations [5]. In our truncation they are given by $C=v=y=0$ and by solving the remaining equations $\partial_{t} C=\partial_{t} v=\partial_{t} y=0$. The latter equations determine the stationary values for $A, B, u, w, s, z$ only incompletely. The present work clearly establishes numerically that these fixed points differ, in general, from the one corresponding to thermal equilibrium. They also prove stable with respect to

\footnotetext{
${ }^{5}$ The infinite volume behavior $\left(<E^{2}>-<E>^{2}\right) /<E^{2}>\sim l^{-1}$ holds for a wide class of $b(\epsilon)$ if its deviation from a constant scales properly with $l$.

${ }^{6}$ By "strict thermalization" we mean an asymptotic approach of the probability distribution to the Boltzmann distribution, the distribution of the microcanonical ensemble.

${ }^{7}$ The general problem with ergodicity arguments is that only a finite neighborhood of a given point in phase space will be reached by an arbitrary trajectory after a finite lapse of time. This is not enough since even very close trajectories typically separate substantially at later time.
} 
small fluctuations. By investigating initial conditions with the same $<L^{2}>$ as for thermal equilibrium we also establish explicitly that the fixed points are not not fully specified by $\langle E\rangle$ and $\left\langle L^{2}\right\rangle$. Numerically, we actually find a large manifold of different fixed points for given $\langle E\rangle$ and $\left\langle L^{2}\right\rangle$, as suggested by the counting of equations and variables for the general stationary solutions. We also establish that the nonthermal fixed points play a role in the asymptotic dynamics. Nonthermal initial conditions typically result in fluctuations around nonthermal stationary solutions at late time.

One may ask if the existence of these fixed points could not be an artifact of the truncation. Three arguments indicate that this is not the case. First, some coordinates in the fixed point manifold are related to exactly conserved combinations of correlation functions like $\left\langle L^{2}>/ l\right.$. Second, the counting of equations and variables indicates that the dimension of the fixed point manifold further increases once 1PI six-point functions or higher couplings are included. Third, we find similar fixed points for different truncations in next-to-leading order in $1 / N$. The ones approached by a given initial condition are close to each other for small $\lambda / N$ and stay substantially away from the thermal fixed points.

Our investigation of the volume dependence indicates that the fixed point manifold does not shrink to the thermal fixed point in the infinite volume limit. Furthermore, all numerical results suggest that the nontrivial fixed points play indeed a dynamical role. From all this a picture for the asymptotic late time behavior of isolated systems emerges where some features of 
"mesoscopic" dynamics survive even in the infinite volume limit. The initial information is not lost completely as for a thermalizing system. Part of the information survives and specifies the stationary solution around which the system oscillates asymptotically.

We observe that for large enough interactions the deviations from thermal equilibrium are small - typically a few percent for the correlation functions and even less for quantities which involve momentum averages. Part of this can be explained by exact relations which hold for all stationary solutions. As an example, let us consider the condition for a static $<q p>$ correlation

$$
\begin{aligned}
& \frac{1}{2} \partial_{t} \int d x<q_{a}(x) p_{a}(x)>=\frac{1}{2} \int d x<p_{a}(x) p_{a}(x)> \\
& -\frac{1}{2} \int d x\left\{<\partial_{i} q_{a}(x) \partial_{i} q_{a}(x)>+m^{2}<q_{a}(x) q_{a}(x)>\right. \\
& \left.+\frac{\lambda}{2 N}<\left(q_{a}(x) q_{a}(x)\right)^{2}>\right\}=0
\end{aligned}
$$

which relates the kinetic and potential energy (note that they are not equal for interacting systems):

$$
<E_{k i n}>=<E_{\text {pot }}>+\frac{\lambda}{8 N} \int d x<\left(q_{a}(x) q_{a}(x)\right)^{2}>
$$

This is, of course, the thermal relation, but it extends to all other stationary solutions as well. We conjecture that large interacting systems generically show an effective irreversible evolution towards asymptotic oscillations around one of the stationary solutions. Then relations of the type (19) hold asymptotically irrespectively of the initial conditions. This explains the robustness of a large set of asymptotic time averages of correlation functions 
- an important part of the initial information is indeed lost. Conversely, a judgement of precise asymptotic thermalization should not be based on generic relations like Eq.(19), but rather on correlation functions which can differ for two inequivalent fixed points.

The lack of exact thermalization of large interacting systems has consequences for "systems in a heat bath" as well. Indeed, we may consider a subsystem, say $q_{a}(x), p_{a}(x)$ for $|x| \leq l_{0} / 2 \ll l / 2$ and view it as evolving in the "heat bath" consisting of the degrees of freedom with $l_{0} / 2<|x| \leq l / 2$. Can we expect that the subsystem effectively thermalizes even though the large isolated system (subsystem and bath) does not exactly thermalize? This question can be addressed by an investigation of correlation functions for the subsystem, say $<q_{a}(x) q_{a}(y)>_{c}$ with $|x|,|y| \leq l_{0} / 2$, or a convenient smoothened version $\left(k_{0}=\pi / l_{0}\right)$

$$
\begin{gathered}
G_{k_{0}}(x, y)=\frac{1}{N}<q_{a}^{k_{0}}(x) q_{a}^{k_{0}}(y)>_{c}=G(x-y) e^{-\frac{1}{2} k_{0}^{2}\left(x^{2}+y^{2}\right)} \\
q_{a}^{k_{0}}(x)=q_{a}(x) e^{-\frac{1}{2} k_{0}^{2} x^{2}}
\end{gathered}
$$

For a translationally invariant ensemble one has

$$
\begin{aligned}
G_{k_{0}}(x, y)= & \int_{q, q^{\prime}} e^{i\left(q x-q^{\prime} y\right)} G_{k_{0}}\left(q, q^{\prime}\right) \\
G_{k_{0}}\left(q, q^{\prime}\right)= & \left(\frac{2 \pi}{k_{0}^{2}}\right)^{D} \exp \left(-\frac{\left(q-q^{\prime}\right)^{2}}{4 k_{0}^{2}}\right) \cdot \\
& \int_{p} G(p) \exp \left(-\frac{\left(p-\frac{1}{2}\left(q+q^{\prime}\right)\right)^{2}}{k_{0}^{2}}\right)
\end{aligned}
$$


In particular, the Fourier transform of $G_{k_{0}}(x, 0)$ reads

$$
\tilde{G}_{k_{0}}(q)=\int_{q^{\prime}} G_{k_{0}}\left(q, q^{\prime}\right)=(2 \pi)^{D / 2} k_{0}^{-D} \int_{p} G(p) e^{-\frac{(q-p)^{2}}{2 k_{0}^{2}}}
$$

Only for $k_{0} \rightarrow 0$ does this reduce to $G(q)$. For nonzero $k_{0}$ (corresponding to a subsystem), however, $\tilde{G}_{k_{0}}(q)$ involves a momentum averaging with width given by $k_{0}$. Because of dephasing, the momentum averaged two-point function $\tilde{G}_{k_{0}}(q)$ approaches a stationary value much more efficiently than $G(q)$. For large $l_{0}$, and nevertheless $l_{0} \ll l$, it is conceivable that $\tilde{G}_{k_{0}}(q)$ actually reaches asymptotically a stationary value whereas $G(q)$ fluctuates around a stationary value. Nevertheless, the time averaged values of $\tilde{G}_{k_{0}}(q)$ and $G(q)$ are still related by Eq.(23). A nonthermal asymptotic behavior of the time averaged $G(q)$ will manifest itself also in the asymptotic form of $\tilde{G}_{k_{0}}(q)$ if it extends over a momentum range with width larger than $k_{0}$. Only variations of $G(q)$ in small momentum ranges will be washed out. From our present investigation we see no indication that asymptotically $\tilde{G}_{k_{0}}(q)$ reaches precisely its thermal value. We conclude that mesoscopic dynamics may also be of relevance for subsystems which are in thermal contact with a "heat bath". The crucial point here is that the heat bath itself is not precisely thermalizing.

In summary, our investigation indicates that isolated systems roughly thermalize for large time, while some quantitative deviations from thermal equilibrium remain. The "loss of memory of the initial conditions", usually assumed in the picture of thermalization, turns out not to be complete. This holds for interacting systems and in the large volume limit. Our results 
question Boltzmann's thermalization conjecture for isolated systems. They suggest that even large interacting isolated systems do not thermalize in a strict sense.

Our treatment is based on an exact evolution equation for the time dependence of equal time correlation functions. Nevertheless, the solution of these equations involves approximations in the form of a truncation of the time dependent effective action. Since our findings touch the basics of thermodynamics, they should be questioned by an independent method. One possibility seems the numerical solution of the microscopic equation (2) for a large sample of different initial conditions. Taking an ensemble average over the initial conditions gives directly the equal time correlation functions which can be compared with the present work. Such a computation could establish definitely if the findings of this work are substantially affected by the truncation or not.

Acknowledgement: We thank L. Bettencourt for many helpful discussions.

\section{Appendix A: Flow Equations}

In this appendix we present the evolution equation for the 1PI 4-point functions which are not specified in the main text.

$$
\begin{aligned}
\dot{v}\left(q_{1}, q_{2}, q_{3}\right)= & {\left[2 \omega^{2}\left(q_{2}\right)\left(w\left(q_{1}, q_{2}, q_{3}\right)-s\left(q_{1}, q_{2}, q_{3}\right)-s\left(q_{1}, q_{2}, q_{4}\right)\right)\right.} \\
& +2 \omega^{2}\left(q_{3}\right) s\left(q_{1}, q_{3}, q_{2}\right)-4 u\left(q_{1}, q_{2}, q_{3}\right)+4 \lambda B\left(q_{1}\right)-\frac{\gamma\left(q_{1}\right)}{N} v\left(q_{1}, q_{2}, q_{3}\right)
\end{aligned}
$$




$$
\begin{aligned}
& -4 \lambda\left(B\left(q_{1}\right)\left(S_{1}\left(q_{1}+q_{2}, q_{3}\right)+S_{2}\left(q_{1}+q_{3}, q_{2}\right)\right)+C\left(q_{4}\right) S_{3}\left(q_{1}+q_{2}, q_{1}\right)\right. \\
& \left.+2 C\left(q_{2}\right) S_{5}\left(q_{2}+q_{3}, q_{1}\right)+C\left(q_{4}\right) S_{6}\left(q_{1}+q_{3}, q_{1}\right)\right) \\
& \left.-\lambda \hbar^{2} B\left(q_{1}\right)\left(C\left(q_{2}\right) C\left(q_{3}\right)+C\left(q_{3}\right) C\left(q_{4}\right)+C\left(q_{2}\right) C\left(q_{4}\right)\right)\right]_{S Y M} \\
& \dot{w}\left(q_{1}, q_{2}, q_{3}\right)=\left[\omega^{2}\left(q_{3}\right)\left(y\left(q_{1}, q_{2}, q_{3}\right)+y\left(q_{1}, q_{3}, q_{2}\right)+y\left(q_{2}, q_{3}, q_{1}\right)\right)-v\left(q_{1}, q_{2}, q_{3}\right)\right. \\
& -v\left(q_{2}, q_{4}, q_{1}\right)-v\left(q_{2}, q_{3}, q_{1}\right)-\frac{\gamma\left(q_{1}\right)+\gamma\left(q_{2}\right)}{N} w\left(q_{1}, q_{2}, q_{3}\right) \\
& -\lambda\left(C\left(q_{3}\right) S_{4}\left(q_{1}, q_{2}\right)+8 B\left(q_{2}\right) S_{5}\left(q_{2}+q_{3}, q_{1}\right)\right) \\
& -4 \lambda\left(B\left(q_{1}\right) S_{3}\left(-q_{1}-q_{3}, q_{2}\right)+B\left(q_{1}\right) S_{6}\left(q_{2}+q_{3}, q_{2}\right)\right. \\
& \left.\left.+C\left(q_{3}\right) S_{7}\left(q_{1}, q_{2}\right)\right)-3 \lambda \hbar^{2} B\left(q_{1}\right) B\left(q_{2}\right) C\left(q_{3}\right)\right]_{S Y M} \\
& \dot{s}\left(q_{1}, q_{2}, q_{3}\right)=\left[2 \omega^{2}\left(q_{3}\right) y\left(q_{1}, q_{3}, q_{2}\right)-2 v\left(q_{2}, q_{4}, q_{1}\right)-\frac{\gamma\left(q_{1}\right)+\gamma\left(q_{2}\right)}{N} s\left(q_{1}, q_{2}, q_{3}\right)\right. \\
& -4 \lambda\left(B\left(q_{1}\right) S_{3}\left(-q_{1}-q_{3}, q_{2}\right)+B\left(q_{1}\right) S_{6}\left(q_{2}+q_{3}, q_{2}\right)+C\left(q_{3}\right) S_{7}\left(q_{1}, q_{2}\right)\right) \\
& \left.-2 \lambda \hbar^{2} B\left(q_{1}\right) B\left(q_{2}\right) C\left(q_{3}\right)\right]_{S Y M} \\
& \dot{y}\left(q_{1}, q_{2}, q_{3}\right)=\left[4 \omega^{2}\left(q_{4}\right) z\left(q_{1}, q_{2}, q_{3}\right)-2 w\left(q_{1}, q_{2}, q_{3}\right)-s\left(q_{2}, q_{3}, q_{1}\right)-s\left(q_{1}, q_{3}, q_{2}\right)\right. \\
& +s\left(q_{1}, q_{2}, q_{3}\right)+s\left(q_{1}, q_{2}, q_{4}\right)-\frac{\gamma\left(q_{1}\right)+\gamma\left(q_{2}\right)+\gamma\left(q_{3}\right)}{N} y\left(q_{1}, q_{2}, q_{3}\right) \\
& \left.-4 \lambda\left(B\left(q_{3}\right) \frac{S_{4}\left(q_{1}, q_{2}\right)}{4}+B\left(q_{1}\right) S_{7}\left(q_{2}, q_{3}\right)\right)-\lambda \hbar^{2} B\left(q_{1}\right) B\left(q_{2}\right) B\left(q_{3}\right)\right]_{S Y M} \\
& \dot{z}\left(q_{1}, q_{2}, q_{3}\right)=\left[-y\left(q_{1}, q_{2}, q_{3}\right)-4 \frac{\gamma\left(q_{1}\right)}{N} z\left(q_{1}, q_{2}, q_{3}\right)\right]_{S Y M} .
\end{aligned}
$$

They involve the following momentum integrals:

$$
\begin{aligned}
S_{3}\left(q_{1}, q_{2}\right) \equiv & \frac{1}{2 N} \int_{q} G(q) G\left(q-q_{1}\right)\left[\frac{1}{2} v\left(q_{2},-q, q-q_{1}\right)+\frac{1}{2} v\left(q_{2}, q-q_{1},-q\right)\right. \\
& +\frac{N+2}{2} v\left(q_{2}, q_{1}-q_{2},-q\right)+\frac{1}{2} c(q) c\left(q-q_{1}\right)\left((N+2) y\left(q-q_{1},-q, q_{2}\right)\right. \\
& \left.+y\left(q_{2}, q-q_{1},-q\right)+y\left(q_{2},-q, q-q_{1}\right)\right)-c(q) s\left(-q, q_{2}, q-q_{1}\right)
\end{aligned}
$$




$$
\begin{aligned}
& \left.-c\left(q-q_{1}\right)\left(N s\left(q-q_{1}, q_{2},-q\right)+2 w\left(q-q_{1}, q_{2},-q\right)\right)\right] \\
S_{4}\left(q_{1}, q_{2}\right) \equiv & \frac{1}{N} \int_{q} G(q) G\left(q-q_{1}-q_{2}\right) \cdot \\
& {\left[(N+2)\left(w\left(q_{1}, q_{2}, q-q_{1}-q_{2}\right)-\frac{1}{2} s\left(q_{1}, q_{2}, q-q_{1}-q_{2}\right)-\frac{1}{2} s\left(q_{1}, q_{2},-q\right)\right)\right.} \\
& +s\left(q_{1}, q_{2},-q\right)+2 c\left(q-q_{1}-q_{2}\right) c(q)\left(N z\left(q-q_{1}-q_{2},-q, q_{1}\right)\right. \\
& \left.+2 z\left(q_{2}, q-q_{1}-q_{2}, q_{1}\right)+2 z\left(q_{2}, q_{1}, q-q_{1}-q_{2}\right)\right) \\
& \left.-c(q)\left(N y\left(q_{1}, q_{2},-q\right)+2 y\left(-q, q_{1}, q_{2}\right)+2 y\left(q_{1}, q_{2},-q\right)\right)\right] \\
S_{5}\left(q_{1}, q_{2}\right)= & \frac{1}{8 N} \int_{q} G(q) G\left(-q-q_{1}\right) \cdot \\
& {\left[2 v\left(q_{2},-q, q+q_{1}\right)+2 c(q) c\left(q+q_{1}\right) y\left(q_{2},-q, q+q_{1}\right)\right.} \\
& -4 c(q)\left(w\left(-q, q_{2}, q+q_{1}\right)-\frac{1}{2} s\left(-q, q_{2}, q+q_{1}\right)-\frac{1}{2} s\left(-q, q_{2},-q_{1}-q_{2}\right)\right) \\
& \left.-2 c\left(-q-q_{1}\right) s\left(q_{2}, q+q_{1},-q\right)\right] \\
& \frac{1}{2 N} \int_{q} G(q) G\left(-q-q_{1}\right) \cdot \\
S_{7}\left(q_{1}, q_{2}\right)= & \frac{1}{2 N} \int_{q} G\left(q_{2},-q-q_{1}, q\right)-c(q) s\left(q_{2},-q,-q-q_{1}\right)-2 c\left(-q-q_{1}\right)\left(w\left(-q-q_{1}, q_{2}, q\right)\right. \\
& {\left[s\left(q_{2}, q_{1},-q\right)-c(q) y\left(-q, q_{2}, q_{1}\right)-c\left(q-q_{1}-q_{2}\right) y\left(q-q_{1}-q_{2}, q_{1}, q_{2}\right)\right.} \\
& \left.+4 c(q) c\left(q-q_{1}-q_{2}\right) z\left(q_{1}, q-q_{1}-q_{2}, q_{2}\right)\right] . \\
S_{6}\left(q_{1}, q_{2}\right)= & \left.\frac{1}{2} s\left(-q-q_{1}, q_{2}, q\right)-\frac{1}{2} s\left(-q-q_{1}, q_{2}, q_{1}-q_{2}\right)\right) \\
+ & \left.(q) c\left(-q-q_{1}\right) y\left(q_{2},-q_{1}-q, q\right)\right] \\
&
\end{aligned}
$$

\section{References}

[1] L.M.A. Bettencourt and C. Wetterich, hep-ph/9805360. 
[2] C. Wetterich, hep-th/9612206, Phys. Rev. Lett. 78, 3598 (1997).

[3] C. Wetterich, hep-th/9703006, Phys. Rev. E 56, 2687 (1997).

H. Nachbagauer, hep-th/9703018.

[4] J. Yvon, La Théorie Statistique des Fluides et l'Equation d'Etat, Act. scient. et ind. No 203, Hermann, Paris, 1935.

M. Born and H.S. Green, Proc. Roy. Soc. Lond. A188, 10 (1946).

J.G. Kirkwood, J. Chem. Phys. 14, 180 (1946).

N.N. Bogoliubov, J. Phys. USSR 10, 257-265 (1946).

[5] L. M.A. Bettencourt and C. Wetterich, hep-ph/9712429, Phys. Lett. B 430, 140-150 (1998).

[6] F. Cooper, S. Habib, Y. Kluger, and E. Mottola, hep-ph/9610345, Phys. Rev. D 55, 6471 (1997).

S. Habib, Y. Kluger, E. Mottola, and J.P. Paz, hep-ph/9509413, Phys. Rev. Lett. 76, 4460 (1996).

D. Boyanovsky, D. Cormier, H.J. de Vega, and R. Holman, hepph/9401308, Phys. Rev. D 51, 734 (1995). 